\title{
Coefficient Estimates for New Subclasses of Analytic and Bi-Univalent Functions Defined by Al-Oboudi Differential Operator
}

\author{
Serap Bulut \\ Civil Aviation College, Kocaeli University, Arslanbey Campus, İzmit 41285 Kocaeli, Turkey \\ Correspondence should be addressed to Serap Bulut; bulutserap@yahoo.com
}

Received 7 May 2013; Accepted 4 October 2013

Academic Editor: Stanislav Hencl

Copyright (C) 2013 Serap Bulut. This is an open access article distributed under the Creative Commons Attribution License, which permits unrestricted use, distribution, and reproduction in any medium, provided the original work is properly cited.

We introduce and investigate two new subclasses $\mathcal{N}_{\Sigma}^{\delta, \mu}(n, \alpha, \lambda)$ and $\mathcal{N}_{\Sigma}^{\delta, \mu}(n, \beta, \lambda)$ of analytic and bi-univalent functions in the open unit disk $\mathbb{U}$. For functions belonging to these classes, we obtain estimates on the first two Taylor-Maclaurin coefficients $\left|a_{2}\right|$ and $\left|a_{3}\right|$.

\section{Introduction}

Let $\mathbb{R}=(-\infty, \infty)$ be the set of real numbers, $\mathbb{C}$ the set of complex numbers, and

$$
\mathbb{N}:=\{1,2,3, \ldots\}=\mathbb{N}_{0} \backslash\{0\}
$$

the set of positive integers.

Let $\mathscr{A}$ denote the class of all functions of the form

$$
f(z)=z+\sum_{k=2}^{\infty} a_{k} z^{k}
$$

which are analytic in the open unit disk

$$
\mathbb{U}=\{z: z \in \mathbb{C},|z|<1\} .
$$

We also denote by $\mathcal{S}$ the class of all functions in the normalized analytic function class $\mathscr{A}$ which are univalent in $\mathbb{U}$.

For $f \in \mathscr{A}$, Al-Oboudi [1] introduced the following operator:

$$
\begin{gathered}
D_{\delta}^{0} f(z)=f(z), \\
D_{\delta}^{1} f(z)=(1-\delta) f(z)+\delta z f^{\prime}(z) \\
=: D_{\delta} f(z) \quad(\delta \geq 0) \\
D_{\delta}^{n} f(z)=D_{\delta}\left(D_{\delta}^{n-1} f(z)\right) \quad(n \in \mathbb{N}) .
\end{gathered}
$$

If $f$ is given by (2), then from (5) and (6) we see that

$$
D_{\delta}^{n} f(z)=z+\sum_{k=2}^{\infty}[1+(k-1) \delta]^{n} a_{k} z^{k} \quad\left(n \in \mathbb{N}_{0}\right)
$$

with $D_{\delta}^{n} f(0)=0$. When $\delta=1$, we get Sălăgean's differential operator [2].

Since univalent functions are one-to-one, they are invertible and the inverse functions need not be defined on the entire unit disk $\mathbb{U}$. In fact, the Koebe one-quarter theorem [3] ensures that the image of $\mathbb{U}$ under every univalent function $f \in \mathcal{S}$ contains a disk of radius $1 / 4$. Thus, every function $f \in \mathscr{A}$ has an inverse $f^{-1}$, which is defined by

$$
\begin{gathered}
f^{-1}(f(z))=z \quad(z \in \mathbb{U}), \\
f\left(f^{-1}(w)\right)=w \quad\left(|w|<r_{0}(f) ; r_{0}(f) \geq \frac{1}{4}\right) .
\end{gathered}
$$

In fact, the inverse function $f^{-1}$ is given by

$$
\begin{aligned}
f^{-1}(w)= & w-a_{2} w^{2}+\left(2 a_{2}^{2}-a_{3}\right) w^{3} \\
& -\left(5 a_{2}^{3}-5 a_{2} a_{3}+a_{4}\right) w^{4}+\cdots .
\end{aligned}
$$

A function $f \in \mathscr{A}$ is said to be bi-univalent in $\mathbb{U}$ if both $f$ and $f^{-1}$ are univalent in $\mathbb{U}$. Let $\Sigma$ denote the class of biunivalent functions in $\mathbb{U}$ given by (2). For a brief history and 
interesting examples of functions in the class $\Sigma$, see [4] (see also $[5,6])$. In fact, the aforecited work of Srivastava et al. [4] essentially revived the investigation of various subclasses of the bi-univalent function class $\Sigma$ in recent years; it was followed by such works as those by Frasin and Aouf [7], Çăglar et al. [8], Porwal and Darus [9], and others (see, for example, [10-13]).

The object of the present paper is to introduce two new subclasses of the function class $\Sigma$ and find estimates on the coefficients $\left|a_{2}\right|$ and $\left|a_{3}\right|$ for functions in these new subclasses of the function class $\Sigma$.

Firstly, in order to derive our main results, we need the following lemma.

Lemma 1 (see [14]). If $p \in \mathscr{P}$, then $\left|c_{k}\right| \leq 2$ for each $k$, where $\mathscr{P}$ is the family of all functions $p$ analytic in $\mathbb{U}$ for which

$$
\Re(p(z))>0, \quad p(z)=1+c_{1} z+c_{2} z^{2}+\cdots
$$

for $z \in \mathbb{U}$.

\section{Coefficient Bounds for the Function Class $\mathcal{N}_{\Sigma}^{\delta, \mu}(n, \alpha, \lambda)$}

Definition 2. A function $f(z)$ given by (2) is said to be in the class $\mathcal{N}_{\Sigma}^{\delta, \mu}(n, \alpha, \lambda)$ if the following conditions are satisfied:

$$
\begin{gathered}
f \in \Sigma, \\
\mid \arg \left((1-\lambda)\left(\frac{D_{\delta}^{n} f(z)}{z}\right)^{\mu}\right. \\
\left.+\lambda\left(D_{\delta}^{n} f(z)\right)^{\prime}\left(\frac{D_{\delta}^{n} f(z)}{z}\right)^{\mu-1}\right) \mid<\frac{\alpha \pi}{2} \\
(0<\alpha \leq 1, \lambda \geq 1, \mu \geq 0, \\
\mid \arg \left((1-\lambda)\left(\frac{D_{\delta}^{n} g(w)}{w}\right)^{\mu}, n \in \mathbb{N}_{0}, z \in \mathbb{U}\right), \\
\left.+\lambda\left(D_{\delta}^{n} g(w)\right)^{\prime}\left(\frac{D_{\delta}^{n} g(w)}{w}\right)^{\mu-1}\right) \mid<\frac{\alpha \pi}{2} \\
(0<\alpha \leq 1, \lambda \geq 1, \mu \geq 0, \\
\left.\delta \geq 0, n \in \mathbb{N}_{0}, w \in \mathbb{U}\right),
\end{gathered}
$$

where the function $g$ is given by

$$
\begin{aligned}
g(w)= & w-a_{2} w^{2}+\left(2 a_{2}^{2}-a_{3}\right) w^{3} \\
& -\left(5 a_{2}^{3}-5 a_{2} a_{3}+a_{4}\right) w^{4}+\cdots
\end{aligned}
$$

and $D_{\delta}^{n}$ is the Al-Oboudi differential operator.
Remark 3. In Definition 2, if we choose

(i) $n=0$, then we have the class

$$
\mathcal{N}_{\Sigma}^{\delta, \mu}(0, \alpha, \lambda)=\mathcal{N}_{\Sigma}^{\mu}(\alpha, \lambda)
$$

introduced by Çağlar et al. [8];

(ii) $n=0$ and $\mu=1$, then we have the class

$$
\mathcal{N}_{\Sigma}^{\delta, 1}(0, \alpha, \lambda)=\mathscr{B}_{\Sigma}(\alpha, \lambda)
$$

introduced by Frasin and Aouf [7];

(iii) $n=0, \mu=1$, and $\lambda=1$, then we have the class

$$
\mathcal{N}_{\Sigma}^{\delta, 1}(0, \alpha, 1)=\mathscr{H}_{\Sigma}^{\alpha}
$$

introduced by Srivastava et al. [4];

(iv) $n=0, \mu=0$, and $\lambda=1$, then we have the class

$$
\mathcal{N}_{\Sigma}^{\delta, 0}(0, \alpha, 1)=\mathcal{S}_{\Sigma}^{*}[\alpha]
$$

of strongly bi-starlike functions of order $\alpha$, introduced by Brannan and Taha $[5,6]$;

(v) $\delta=1$ and $\mu=1$, then we have the class

$$
\mathscr{N}_{\Sigma}^{1,1}(n, \alpha, \lambda)=\mathscr{B}_{\Sigma}(n, \alpha, \lambda)
$$

introduced by Porwal and Darus [9].

Theorem 4. Let the function $f(z)$ given by the TaylorMaclaurin series expansion (2) be in the function class

$$
\mathcal{N}_{\Sigma}^{\delta, \mu}(n, \alpha, \lambda) \quad\left(0<\alpha \leq 1, \lambda \geq 1, \mu \geq 0, \delta \geq 0, n \in \mathbb{N}_{0}\right)
$$

Then,

$$
\begin{aligned}
& \left|a_{2}\right| \leq(2 \alpha) \\
& \times\left(\left((1+\delta)^{2 n}(\lambda+\mu)^{2}\right.\right. \\
& +\alpha\left[2(1+2 \delta)^{n}(2 \lambda+\mu)\right. \\
& \left.\left.\left.\quad-(1+\delta)^{2 n}\left(\lambda^{2}+2 \lambda+\mu\right)\right]\right)^{1 / 2}\right)^{-1}
\end{aligned}
$$

$$
\left|a_{3}\right| \leq \frac{4 \alpha^{2}}{(1+\delta)^{2 n}(\lambda+\mu)^{2}}+\frac{2 \alpha}{(1+2 \delta)^{n}(2 \lambda+\mu)} \text {. }
$$

Proof. First of all, it follows from conditions (11) that

$$
(1-\lambda)\left(\frac{D_{\delta}^{n} f(z)}{z}\right)^{\mu}+\lambda\left(D_{\delta}^{n} f(z)\right)^{\prime}\left(\frac{D_{\delta}^{n} f(z)}{z}\right)^{\mu-1}
$$




$$
\begin{aligned}
&=[p(z)]^{\alpha} \quad(z \in \mathbb{U}), \\
&(1-\lambda)\left(\frac{D_{\delta}^{n} g(w)}{w}\right)^{\mu}+\lambda\left(D_{\delta}^{n} g(w)\right)^{\prime}\left(\frac{D_{\delta}^{n} g(w)}{w}\right)^{\mu-1} \\
&=[q(w)]^{\alpha} \quad(w \in \mathbb{U}),
\end{aligned}
$$

respectively, where

$$
\begin{aligned}
& p(z)=1+p_{1} z+p_{2} z^{2}+\cdots, \\
& q(w)=1+q_{1} w+q_{2} w^{2}+\cdots
\end{aligned}
$$

in $\mathscr{P}$. Now, upon equating the coefficients in (21), we get

$$
\begin{gathered}
(1+\delta)^{n}(\lambda+\mu) a_{2}=\alpha p_{1}, \\
(1+2 \delta)^{n}(2 \lambda+\mu) a_{3}+(1+\delta)^{2 n}(\mu-1)\left(\lambda+\frac{\mu}{2}\right) a_{2}^{2} \\
=\alpha p_{2}+\frac{\alpha(\alpha-1)}{2} p_{1}^{2} \\
-(1+\delta)^{n}(\lambda+\mu) a_{2}=\alpha q_{1}, \\
-(1+2 \delta)^{n}(2 \lambda+\mu) a_{3}+\left[4(1+2 \delta)^{n}+(1+\delta)^{2 n}(\mu-1)\right] \\
\times\left(\lambda+\frac{\mu}{2}\right) a_{2}^{2}=\alpha q_{2}+\frac{\alpha(\alpha-1)}{2} q_{1}^{2} .
\end{gathered}
$$

From (23) and (25), we obtain

$$
\begin{gathered}
p_{1}=-q_{1}, \\
2(1+\delta)^{2 n}(\lambda+\mu)^{2} a_{2}^{2}=\alpha^{2}\left(p_{1}^{2}+q_{1}^{2}\right) .
\end{gathered}
$$

Also, from (24), (26), and (28), we find that

$$
\begin{aligned}
& {\left[2(1+2 \delta)^{n}+(1+\delta)^{2 n}(\mu-1)\right](2 \lambda+\mu) a_{2}^{2}} \\
& \quad=\alpha\left(p_{2}+q_{2}\right)+\frac{\alpha(\alpha-1)}{2}\left(p_{1}^{2}+q_{1}^{2}\right) \\
& \quad=\alpha\left(p_{2}+q_{2}\right)+\frac{\alpha-1}{\alpha}(1+\delta)^{2 n}(\lambda+\mu)^{2} a_{2}^{2} .
\end{aligned}
$$

Therefore, we obtain

$$
\begin{aligned}
a_{2}^{2}= & \left(\alpha^{2}\left(p_{2}+q_{2}\right)\right) \\
& \times\left(2 \alpha(1+2 \delta)^{n}(2 \lambda+\mu)\right. \\
& \left.\quad+(1+\delta)^{2 n}\left[(\lambda+\mu)^{2}-\alpha\left(\lambda^{2}+2 \lambda+\mu\right)\right]\right)^{-1} .
\end{aligned}
$$

Applying Lemma 1 for the aforementioned equality, we get the desired estimate on the coefficient $\left|a_{2}\right|$ as asserted in (19).

Next, in order to find the bound on the coefficient $\left|a_{3}\right|$, we subtract (26) from (24). We thus get

$$
\begin{aligned}
2(1+2 \delta)^{n}(2 \lambda+\mu) a_{3}-2(1+2 \delta)^{n}(2 \lambda+\mu) a_{2}^{2} \\
=\alpha\left(p_{2}-q_{2}\right)+\frac{\alpha(\alpha-1)}{2}\left(p_{1}^{2}-q_{1}^{2}\right) .
\end{aligned}
$$

It follows from (27), (28), and (31) that

$$
a_{3}=\frac{\alpha^{2}\left(p_{1}^{2}+q_{1}^{2}\right)}{2(1+\delta)^{2 n}(\lambda+\mu)^{2}}+\frac{\alpha\left(p_{2}-q_{2}\right)}{2(1+2 \delta)^{n}(2 \lambda+\mu)} .
$$

Applying Lemma 1 for the previous equality, we get the desired estimate on the coefficient $\left|a_{3}\right|$ as asserted in (20).

If we take $n=0$ in Theorem 4 , then we have the following corollary.

Corollary 5 (see [8]). Let the function $f(z)$ given by the Taylor-Maclaurin series expansion (2) be in the class $\mathcal{N}_{\Sigma}^{\mu}(\alpha, \lambda)(0<\alpha \leq 1, \lambda \geq 1, \mu \geq 0)$. Then,

$$
\begin{gathered}
\left|a_{2}\right| \leq \frac{2 \alpha}{\sqrt{(\lambda+\mu)^{2}+\alpha\left(\mu+2 \lambda-\lambda^{2}\right)}}, \\
\left|a_{3}\right| \leq \frac{4 \alpha^{2}}{(\lambda+\mu)^{2}}+\frac{2 \alpha}{2 \lambda+\mu} .
\end{gathered}
$$

If we take $n=0$ and $\mu=1$ in Theorem 4 , then we have the following corollary.

Corollary 6 (see [7]). Let the function $f(z)$ given by the Taylor-Maclaurin series expansion (2) be in the class $\mathscr{B}_{\Sigma}(\alpha, \lambda)(0<\alpha \leq 1, \lambda \geq 1)$. Then

$$
\begin{gathered}
\left|a_{2}\right| \leq \frac{2 \alpha}{\sqrt{(\lambda+1)^{2}+\alpha\left(1+2 \lambda-\lambda^{2}\right)}}, \\
\left|a_{3}\right| \leq \frac{4 \alpha^{2}}{(\lambda+1)^{2}}+\frac{2 \alpha}{2 \lambda+1} .
\end{gathered}
$$

If we take $n=0, \mu=1$ and $\lambda=1$ in Theorem 4 , then we have the following corollary.

Corollary 7 (see [4]). Let the function $f(z)$ given by the Taylor-Maclaurin series expansion (2) be in the class $\mathscr{H}_{\Sigma}^{\alpha}(0<$ $\alpha \leq 1)$. Then,

$$
\begin{aligned}
& \left|a_{2}\right| \leq \alpha \sqrt{\frac{2}{\alpha+2}}, \\
& \left|a_{3}\right| \leq \frac{\alpha(3 \alpha+2)}{3} .
\end{aligned}
$$

If we take $n=0, \mu=0$, and $\lambda=1$ in Theorem 4 , then we have the following corollary.

Corollary 8. Let the function $f(z)$ given by the TaylorMaclaurin series expansion (2) be in the class $\mathcal{S}_{\Sigma}^{*}[\alpha](0<\alpha \leq$ 1). Then,

$$
\begin{aligned}
& \left|a_{2}\right| \leq \frac{2 \alpha}{\sqrt{1+\alpha}}, \\
& \left|a_{3}\right| \leq 4 \alpha^{2}+\alpha .
\end{aligned}
$$

If we take $\delta=1$ and $\mu=1$ in Theorem 4 , then we have the following corollary. 
Corollary 9 (see [9]). Let the function $f(z)$ given by the Taylor-Maclaurin series expansion (2) be in the function class $\mathscr{B}_{\Sigma}(n, \alpha, \lambda)\left(0<\alpha \leq 1, \lambda \geq 1, n \in \mathbb{N}_{0}\right)$. Then,

$\left|a_{2}\right|$

$$
\begin{gathered}
\leq \frac{2 \alpha}{\sqrt{4^{n}(\lambda+1)^{2}+\alpha\left[2.3^{n}(2 \lambda+1)-4^{n}(\lambda+1)^{2}\right]}}, \\
\left|a_{3}\right| \leq \frac{4 \alpha^{2}}{4^{n}(\lambda+1)^{2}}+\frac{2 \alpha}{3^{n}(2 \lambda+1)} .
\end{gathered}
$$

\section{Coefficient Bounds for the Function Class $\mathcal{N}_{\Sigma}^{\delta, \mu}(n, \beta, \lambda)$}

Definition 10. A function $f(z)$ given by (2) is said to be in the class $\mathcal{N}_{\Sigma}^{\delta, \mu}(n, \beta, \lambda)$ if the following conditions are satisfied:

$$
\begin{gathered}
f \in \Sigma, \\
\Re\left\{(1-\lambda)\left(\frac{D_{\delta}^{n} f(z)}{z}\right)^{\mu}\right. \\
\left.+\lambda\left(D_{\delta}^{n} f(z)\right)^{\prime}\left(\frac{D_{\delta}^{n} f(z)}{z}\right)^{\mu-1}\right\}>\beta \\
(0 \leq \beta<1, \lambda \geq 1, \mu \geq 0, \\
\Re\left\{(1-\lambda)\left(\frac{D_{\delta}^{n} g(w)}{w}\right)^{\mu}, n \in \mathbb{N}_{0}, z \in \mathbb{U}\right), \\
\left.+\lambda\left(D_{\delta}^{n} g(w)\right)^{\prime}\left(\frac{D_{\delta}^{n} g(w)}{w}\right)^{\mu-1}\right\}>\beta \\
(0 \leq \beta<1, \lambda \geq 1, \mu \geq 0, \\
\left.\delta \geq 0, n \in \mathbb{N}_{0}, w \in \mathbb{U}\right),
\end{gathered}
$$

where the function $g$ is defined by (12) and $D_{\delta}^{n}$ is the AlOboudi differential operator.

Remark 11. In Definition 10, if we choose

(i) $n=0$, then we have the class

$$
\mathscr{N}_{\Sigma}^{\delta, \mu}(0, \beta, \lambda)=\mathcal{N}_{\Sigma}^{\mu}(\beta, \lambda)
$$

introduced by Çağlar et al. [8];

(ii) $n=0$ and $\mu=1$, then we have the class

$$
\mathscr{N}_{\Sigma}^{\delta, 1}(0, \beta, \lambda)=\mathscr{B}_{\Sigma}(\beta, \lambda)
$$

introduced by Frasin and Aouf [7];

(iii) $n=0, \mu=1$, and $\lambda=1$, then we have the class

$$
\mathcal{N}_{\Sigma}^{\delta, 1}(0, \beta, 1)=\mathscr{H}_{\Sigma}(\beta)
$$

introduced by Srivastava et al. [4]; (iv) $n=0, \mu=0$, and $\lambda=1$, then we have the class

$$
\mathcal{N}_{\Sigma}^{\delta, 0}(0, \beta, 1)=\mathcal{S}_{\Sigma}^{*}(\beta)
$$

of bi-starlike functions of order $\beta$, introduced by Brannan and Taha $[5,6]$;

(v) $\delta=1$ and $\mu=1$, then we have the class

$$
\mathcal{N}_{\Sigma}^{1,1}(n, \beta, \lambda)=\mathscr{H}_{\Sigma}(n, \beta, \lambda)
$$

introduced by Porwal and Darus [9].

Theorem 12. Let the function $f(z)$ given by the TaylorMaclaurin series expansion (2) be in the function class

$$
\begin{aligned}
& \mathcal{N}_{\Sigma}^{\delta, \mu}(n, \beta, \lambda) \\
& \quad\left(0<\alpha \leq 1, \lambda \geq 1, \mu \geq 0, \delta \geq 0, n \in \mathbb{N}_{0}\right) .
\end{aligned}
$$

Then,

$$
\begin{aligned}
&\left|a_{2}\right| \leq \min \left\{\frac{2(1-\beta)}{(1+\delta)^{n}(\lambda+\mu)},\right.((4(1-\beta)) \\
& \times\left(\left|2(1+2 \delta)^{n}+(1+\delta)^{2 n}(\mu-1)\right|\right. \\
&\left.\left.\times(2 \lambda+\mu))^{-1}\right)^{1 / 2}\right\} \\
&\left|a_{3}\right| \leq \min \left\{\frac{4(1-\beta)^{2}}{(1+\delta)^{2 n}(\lambda+\mu)^{2}}+\frac{2(1-\beta)}{(1+2 \delta)^{n}(2 \lambda+\mu)},\right. \\
& \\
&\left(( 1 - \beta ) \left[\left|4(1+2 \delta)^{n}+(1+\delta)^{2 n}(\mu-1)\right|\right.\right. \\
&\left.\left.\quad+(1+\delta)^{2 n}|\mu-1|\right]\right) \\
& \times\left((1+2 \delta)^{n}\left|2(1+2 \delta)^{n}+(1+\delta)^{2 n}(\mu-1)\right|\right. \\
&\left.\times(2 \lambda+\mu))^{-1}\right\} .
\end{aligned}
$$

Proof. First of all, it follows from conditions (38) that

$$
\begin{aligned}
& (1-\lambda)\left(\frac{D_{\delta}^{n} f(z)}{z}\right)^{\mu}+\lambda\left(D_{\delta}^{n} f(z)\right)^{\prime}\left(\frac{D_{\delta}^{n} f(z)}{z}\right)^{\mu-1} \\
& =\beta+(1-\beta) p(z) \quad(z \in \mathbb{U}), \\
& (1-\lambda)\left(\frac{D_{\delta}^{n} g(w)}{w}\right)^{\mu}+\lambda\left(D_{\delta}^{n} g(w)\right)^{\prime}\left(\frac{D_{\delta}^{n} g(w)}{w}\right)^{\mu-1} \\
& =\beta+(1-\beta) q(w) \quad(w \in \mathbb{U}),
\end{aligned}
$$

respectively, where

$$
\begin{aligned}
& p(z)=1+p_{1} z+p_{2} z^{2}+\cdots, \\
& q(w)=1+q_{1} w+q_{2} w^{2}+\cdots
\end{aligned}
$$


in $\mathscr{P}$. Now, upon equating the coefficients in (47), we get

$$
\begin{gathered}
(1+\delta)^{n}(\lambda+\mu) a_{2}=(1-\beta) p_{1}, \\
(1+2 \delta)^{n}(2 \lambda+\mu) a_{3} \\
+(1+\delta)^{2 n}(\mu-1)\left(\lambda+\frac{\mu}{2}\right) a_{2}^{2}=(1-\beta) p_{2}, \\
-(1+\delta)^{n}(\lambda+\mu) a_{2}=(1-\beta) q_{1}, \\
-(1+2 \delta)^{n}(2 \lambda+\mu) a_{3} \\
+\left[4(1+2 \delta)^{n}+(1+\delta)^{2 n}(\mu-1)\right] \\
\times\left(\lambda+\frac{\mu}{2}\right) a_{2}^{2}=(1-\beta) q_{2} .
\end{gathered}
$$

From (49) and (51), we obtain

$$
\begin{gathered}
p_{1}=-q_{1}, \\
2(1+\delta)^{2 n}(\lambda+\mu)^{2} a_{2}^{2}=(1-\beta)^{2}\left(p_{1}^{2}+q_{1}^{2}\right) .
\end{gathered}
$$

Also, from (50) and (52), we have

$$
\begin{aligned}
& {\left[2(1+2 \delta)^{n}+(1+\delta)^{2 n}(\mu-1)\right]} \\
& \quad \times(2 \lambda+\mu) a_{2}^{2}=(1-\beta)\left(p_{2}+q_{2}\right) .
\end{aligned}
$$

Therefore, from equalities (54) and (55) we find that

$$
\begin{gathered}
\left|a_{2}\right|^{2} \leq \frac{(1-\beta)^{2}\left(\left|p_{1}\right|^{2}+\left|q_{1}\right|^{2}\right)}{2(1+\delta)^{2 n}(\lambda+\mu)^{2}}, \\
\left|a_{2}\right|^{2} \\
\leq \frac{(1-\beta)\left(\left|p_{2}\right|+\left|q_{2}\right|\right)}{\left|2(1+2 \delta)^{n}+(1+\delta)^{2 n}(\mu-1)\right|(2 \lambda+\mu)},
\end{gathered}
$$

respectively, and applying Lemma 1, we get the desired estimate on the coefficient $\left|a_{2}\right|$ as asserted in (45).

Next, in order to find the bound on the coefficient $\left|a_{3}\right|$, we subtract (52) from (50). We thus get

$$
\begin{aligned}
& 2(1+2 \delta)^{n}(2 \lambda+\mu) a_{3} \\
& -2(1+2 \delta)^{n}(2 \lambda+\mu) a_{2}^{2}=(1-\beta)\left(p_{2}-q_{2}\right),
\end{aligned}
$$

which, upon substitution of the value of $a_{2}^{2}$ from (54), yields

$$
\begin{aligned}
a_{3}= & \frac{(1-\beta)^{2}\left(p_{1}^{2}+q_{1}^{2}\right)}{2(1+\delta)^{2 n}(\lambda+\mu)^{2}} \\
& +\frac{(1-\beta)\left(p_{2}-q_{2}\right)}{2(1+2 \delta)^{n}(2 \lambda+\mu)} .
\end{aligned}
$$

On the other hand, by using the (55) into (57), it follows that

$$
\begin{gathered}
a_{3}=(1-\beta) \\
\times\left(2(1+2 \delta)^{n}\left[2(1+2 \delta)^{n}+(1+\delta)^{2 n}(\mu-1)\right]\right. \\
\times(2 \lambda+\mu))^{-1} \times\left\{\left[4(1+2 \delta)^{n}+(1+\delta)^{2 n}(\mu-1)\right] p_{2}\right. \\
\left.-(1+\delta)^{2 n}(\mu-1) q_{2}\right\} .
\end{gathered}
$$

Applying Lemma 1 for (58) and (59), we get the desired estimate on the coefficient $\left|a_{3}\right|$ as asserted in (46).

If we take $n=0$ in Theorem 12, then we have the following corollary.

Corollary 13 (see [8]). Let the function $f(z)$ given by the Taylor-Maclaurin series expansion (2) be in the class $\mathcal{N}_{\Sigma}^{\mu}(\beta, \lambda)(0 \leq \beta<1, \lambda \geq 1, \mu \geq 0)$. Then,

$$
\left|a_{2}\right| \leq \min \left\{\sqrt{\frac{4(1-\beta)}{(\mu+1)(2 \lambda+\mu)}}, \frac{2(1-\beta)}{\lambda+\mu}\right\},
$$

$\left|a_{3}\right|$

$$
\leq\left\{\begin{array}{l}
\min \left\{\frac{4(1-\beta)}{(\mu+1)(2 \lambda+\mu)}, \frac{4(1-\beta)^{2}}{(\lambda+\mu)^{2}}+\frac{2(1-\beta)}{2 \lambda+\mu}\right\}, \\
0 \leq \mu<1 \\
\frac{2(1-\beta)}{2 \lambda+\mu}, \\
\mu \geq 1 .
\end{array}\right.
$$

If we take $n=0$ and $\mu=1$ in Theorem 12, then we have the following corollary.

Corollary 14. Let the function $f(z)$ given by the TaylorMaclaurin series expansion (2) be in the class $\mathscr{B}_{\Sigma}(\beta, \lambda)(0 \leq$ $\beta<1, \lambda \geq 1$ ). Then,

$$
\begin{gathered}
\left|a_{2}\right| \leq \min \left\{\sqrt{\left.\frac{2(1-\beta)}{2 \lambda+1}, \frac{2(1-\beta)}{\lambda+1}\right\},}\right. \\
\left|a_{3}\right| \leq \frac{2(1-\beta)}{2 \lambda+1} .
\end{gathered}
$$

Remark 15. Corollary 14 provides an improvement of the following estimates obtained by Frasin and Aouf [7].

Corollary 16 (see [7]). Let the function $f(z)$ given by the Taylor-Maclaurin series expansion (2) be in the class $\mathscr{B}_{\Sigma}(\beta, \lambda)(0 \leq \beta<1, \lambda \geq 1)$. Then,

$$
\begin{gathered}
\left|a_{2}\right| \leq \sqrt{\frac{2(1-\beta)}{2 \lambda+1}}, \\
\left|a_{3}\right| \leq \frac{4(1-\beta)^{2}}{(\lambda+1)^{2}}+\frac{2(1-\beta)}{2 \lambda+1} .
\end{gathered}
$$


If we take $n=0, \mu=1$, and $\lambda=1$ in Theorem 12 , then we have the following corollary.

Corollary 17. Let the function $f(z)$ given by the TaylorMaclaurin series expansion (2) be in the class $\mathscr{H}_{\Sigma}(\beta)(0 \leq \beta<$ 1). Then,

$$
\begin{gathered}
\left|a_{2}\right| \leq \begin{cases}\sqrt{\frac{2(1-\beta)}{3}}, & 0 \leq \beta \leq \frac{1}{3} \\
1-\beta, & \frac{1}{3} \leq \beta<1,\end{cases} \\
\left|a_{3}\right| \leq \frac{2(1-\beta)}{3} .
\end{gathered}
$$

Remark 18. Corollary 17 provides an improvement of the following estimates obtained by Srivastava et al. [4].

Corollary 19 (see [4]). Let the function $f(z)$ given by the Taylor-Maclaurin series expansion (2) be in the class $\mathscr{H}_{\Sigma}(\beta)(0 \leq \beta<1)$. Then,

$$
\begin{gathered}
\left|a_{2}\right| \leq \sqrt{\frac{2(1-\beta)}{3}}, \\
\left|a_{3}\right| \leq \frac{(1-\beta)(5-3 \beta)}{3} .
\end{gathered}
$$

If we take $n=0, \mu=0$, and $\lambda=1$ in Theorem 12, then we have the following corollary.

Corollary 20. Let the function $f(z)$ given by the TaylorMaclaurin series expansion (2) be in the class $\mathcal{S}_{\Sigma}^{*}(\beta)(0 \leq \beta<$ 1). Then,

$$
\begin{gathered}
\left|a_{2}\right| \leq \sqrt{2(1-\beta)}, \\
\left|a_{3}\right| \leq \begin{cases}2(1-\beta), & 0 \leq \beta \leq \frac{3}{4} \\
(1-\beta)(5-4 \beta), & \frac{3}{4} \leq \beta<1 .\end{cases}
\end{gathered}
$$

Remark 21. Corollary 20 provides an improvement of the following estimates obtained by Brannan and Taha $[5,6]$ (see also [10, Corollary 3.2]).

Corollary 22 (see $[5,6]$ ). Let the function $f(z)$ given by the Taylor-Maclaurin series expansion (2) be in the class $\mathcal{S}_{\Sigma}^{*}(\beta)(0 \leq \beta<1)$. Then,

$$
\begin{gathered}
\left|a_{2}\right| \leq \sqrt{2(1-\beta)}, \\
\left|a_{3}\right| \leq 2(1-\beta) .
\end{gathered}
$$

If we take $\delta=1$ and $\mu=1$ in Theorem 12, then we have the following corollary.
Corollary 23. Let the function $f(z)$ given by the TaylorMaclaurin series expansion (2) be in the function class $\mathscr{H}_{\Sigma}(n, \beta, \lambda)\left(0<\alpha \leq 1, \lambda \geq 1, n \in \mathbb{N}_{0}\right)$. Then,

$$
\begin{gathered}
\left|a_{2}\right| \leq \min \left\{\frac{2(1-\beta)}{2^{n}(\lambda+1)}, \sqrt{\frac{2(1-\beta)}{3^{n}(2 \lambda+1)}}\right\}, \\
\left|a_{3}\right| \leq \frac{2(1-\beta)}{3^{n}(2 \lambda+1)} .
\end{gathered}
$$

Remark 24. Corollary 23 provides an improvement of the following estimates obtained by Porwal and Darus [9].

Corollary 25 (see [9]). Let the function $f(z)$ given by the Taylor-Maclaurin series expansion (2) be in the function class $\mathscr{H}_{\Sigma}(n, \beta, \lambda)\left(0<\alpha \leq 1, \lambda \geq 1, n \in \mathbb{N}_{0}\right)$. Then,

$$
\begin{gathered}
\left|a_{2}\right| \leq \sqrt{\frac{2(1-\beta)}{3^{n}(2 \lambda+1)}}, \\
\left|a_{3}\right| \leq \frac{4(1-\beta)^{2}}{4^{n}(\lambda+1)^{2}}+\frac{2(1-\beta)}{3^{n}(2 \lambda+1)} .
\end{gathered}
$$

\section{References}

[1] F. M. Al-Oboudi, "On univalent functions defined by a generalized Sălăgean operator," International Journal of Mathematics and Mathematical Sciences, vol. 2004, no. 27, pp. 1429-1436, 2004.

[2] G. Sălăgean, "Subclasses of univalent functions," in Complex Analysis-Fifth Romanian-Finnish Seminar, Part 1 (Bucharest, 1981), vol. 1013 of Lecture Notes in Mathematics, pp. 362-372, Springer, Berlin, Germany, 1983.

[3] P. L. Duren, Univalent Functions, vol. 259 of Grundlehren der Mathematischen Wissenschaften, Springer, New York, NY, USA, 1983.

[4] H. M. Srivastava, A. K. Mishra, and P. Gochhayat, "Certain subclasses of analytic and bi-univalent functions," Applied Mathematics Letters, vol. 23, no. 10, pp. 1188-1192, 2010.

[5] D. A. Brannan and T. S. Taha, "Onsome classes of bi-univalent functions," in Mathematical Analysis and Its Applications, S. M. Mazhar, A. Hamoui, and N. S. Faour, Eds., vol. 3 of KFAS Proceedings Series, pp. 53-60, Pergamon Press (Elsevier Science Limited), Oxford, UK, 1988.

[6] D. A. Brannan and T. S. Taha, "On some classes of bi-univalent functions," Studia Universitatis Babeş-Bolyai. Mathematica, vol. 31, no. 2, pp. 70-77, 1986.

[7] B. A. Frasin and M. K. Aouf, "New subclasses of bi-univalent functions," Applied Mathematics Letters, vol. 24, no. 9, pp. 15691573, 2011.

[8] M. Çağlar, H. Orhan, and N. Yağmur, "Coefficient bounds for new subclasses of bi-univalent functions," Filomat, vol. 27, no. 7, pp. 1165-1171, 2013.

[9] S. Porwal and M. Darus, "On a new subclass of bi-univalent functions," Journal of the Egyptian Mathematical Society, vol. 21, no. 3, pp. 190-193, 2013.

[10] S. Bulut, "Coefficient estimates for a class of analytic and biunivalent functions," Novi Sad Journal of Mathematics, vol. 43, no. 2, pp. 59-65, 2013. 
[11] T. Hayami and S. Owa, "Coefficient bounds for bi-univalent functions," Panamerican Mathematical Journal, vol. 22, no. 4, pp. 15-26, 2012.

[12] Q.-H. Xu, Y.-C. Gui, and H. M. Srivastava, "Coefficient estimates for a certain subclass of analytic and bi-univalent functions," Applied Mathematics Letters, vol. 25, no. 6, pp. 990-994, 2012.

[13] Q.-H. Xu, H.-G. Xiao, and H. M. Srivastava, "A certain general subclass of analytic and bi-univalent functions and associated coefficient estimate problems," Applied Mathematics and Computation, vol. 218, no. 23, pp. 11461-11465, 2012.

[14] Ch. Pommerenke, Univalent Functions, Vandenhoeck \& Ruprecht, Göttingen, Germany, 1975. 


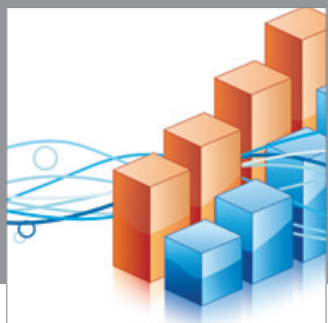

Advances in

Operations Research

mansans

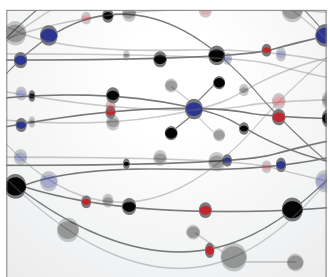

The Scientific World Journal
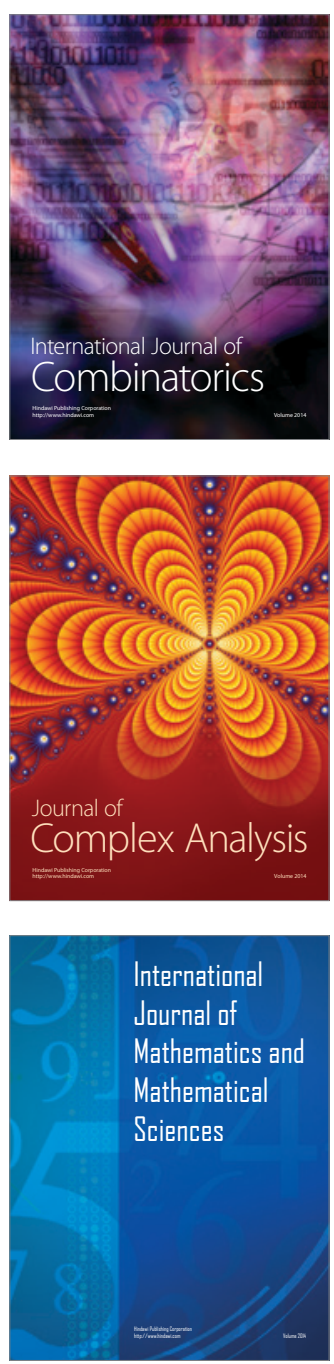
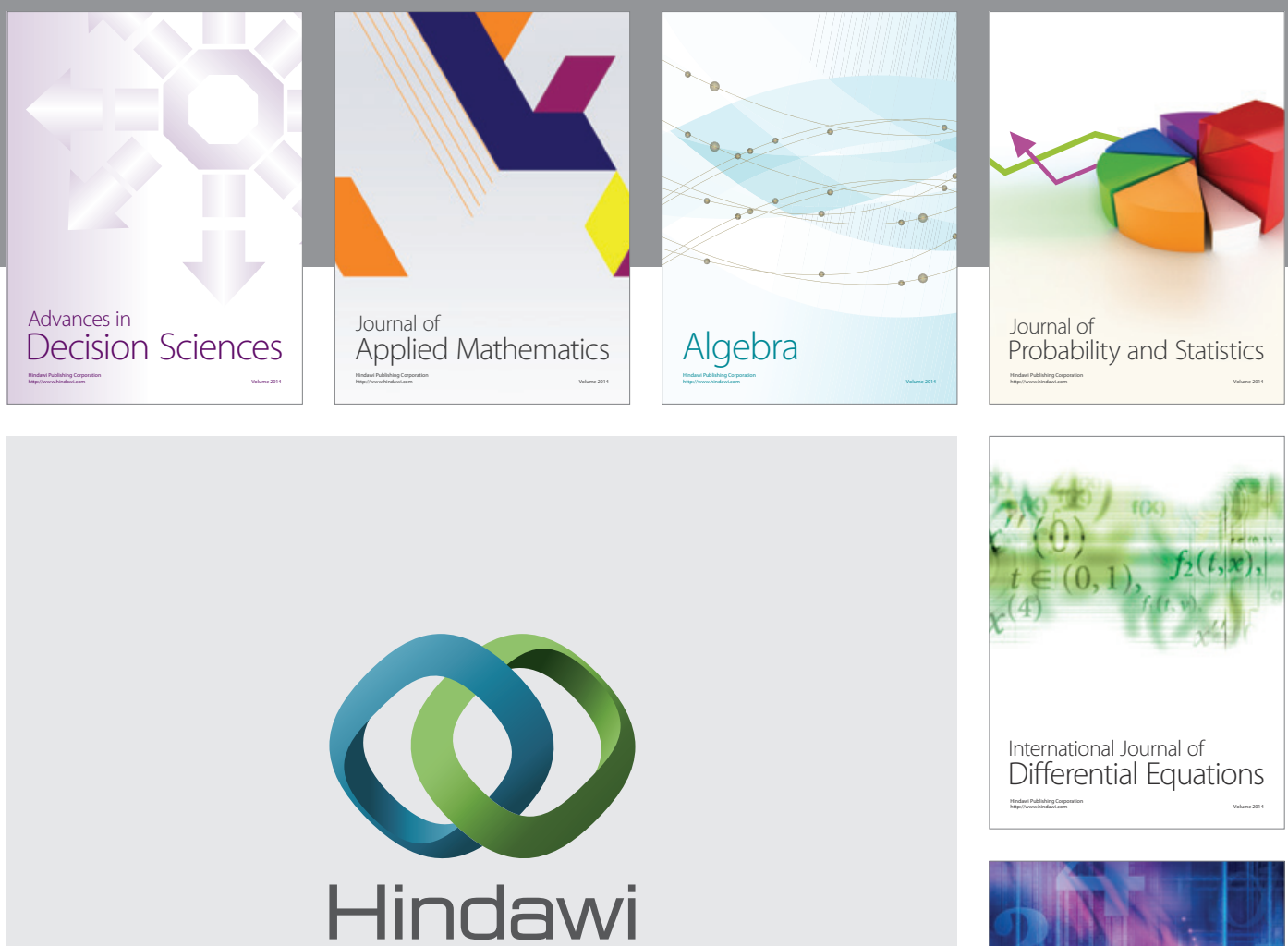

Submit your manuscripts at http://www.hindawi.com
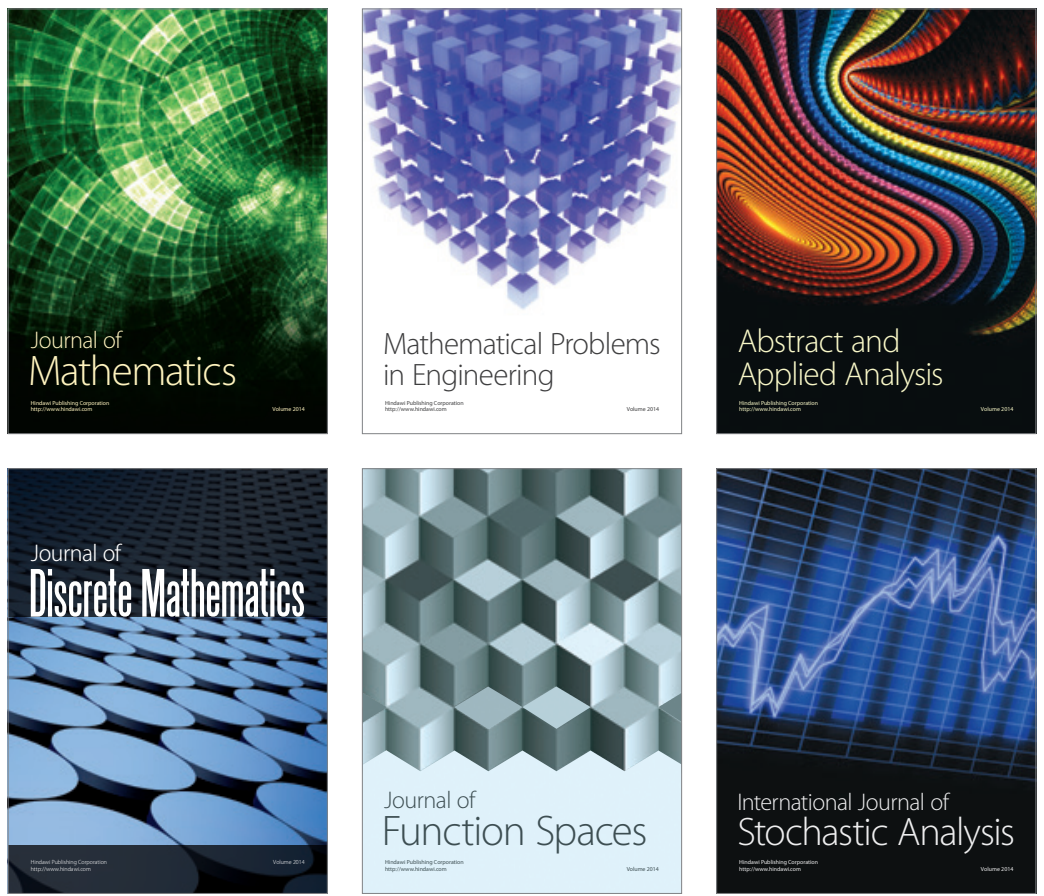

Journal of

Function Spaces

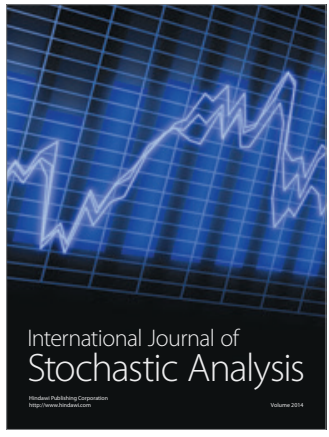

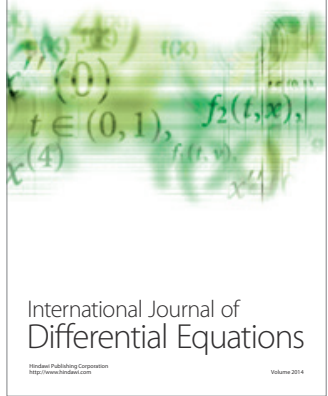
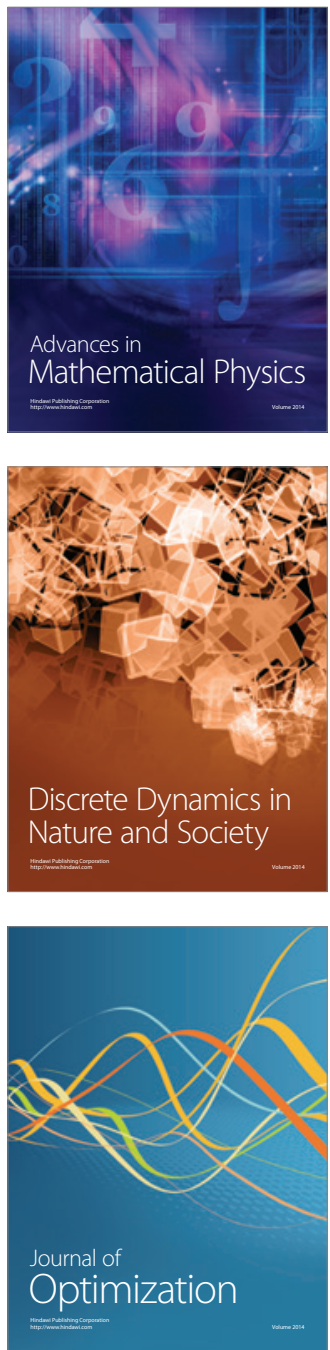